\title{
Spin relaxation times of two-dimensional holes from spin sensitive bleaching of intersubband absorption
}

\author{
Petra Schneider, ${ }^{\text {a) }}$ J. Kainz, S. D. Ganichev, S. N. Danilov, U. Rössler, W. Wegscheider, \\ D. Weiss, and W. Prettl \\ Fakultät Physik, University of Regensburg, 93040 Regensburg, Germany \\ V. V. Bel'kov, M. M. Glazov, and L. E. Golub \\ A. F. Ioffe Physico-Technical Institute, 194021 St. Petersburg, Russia \\ D. Schuh \\ Walter Schottky Institute, Technical University Munich, 85748 Garching, Germany
}

(Received 21 January 2004; accepted 2 April 2004)

\begin{abstract}
We present spin relaxation times of two-dimensional holes obtained by spin sensitive bleaching of the absorption of infrared radiation in $p$-type GaAs/AlGaAs quantum wells (QWs). It is shown that the saturation of intersubband absorption of circularly polarized radiation is mainly controlled by the spin relaxation time of the holes. The saturation behavior has been determined for different QW widths and in a wide range of temperatures with the result that the saturation intensity substantially decreases with narrowing of the QWs. Spin relaxation times are derived from the measured saturation intensities by making use of calculated (linear) absorption coefficients for direct intersubband transitions. It is shown that spin relaxation is due to the D'yakonov-Perel' mechanism governed by hole-hole scattering. The problem of selection rules is addressed. (C) 2004 American Institute of Physics. [DOI: 10.1063/1.1753656]
\end{abstract}

\section{INTRODUCTION}

The spin degree of freedom of charge carriers in semiconductors, of fundamental interest as a dynamic variable, has recently attracted much attention because of its possible role in active spintronic devices. ${ }^{1}$ It is closely related to the polarization degree of freedom of electromagnetic waves by the selection rules which have been used for optical spin orientation. ${ }^{2}$ The spin relaxation times of electrons and holes in semiconductor quantum well structures were measured in time-resolved photoluminescence experiments. ${ }^{3-6}$ In these investigations, which had optical excitation across the band gap, electron-hole pairs are created and the measured spin relaxation times reflect the particular situation of bipolar spin orientation with relaxation processes, in which the electronhole exchange process can play a dominant role. ${ }^{7}$ This situation is not what would be expected in prospective spintronic devices $^{8}$ which are likely to operate with one kind of carrier only, spin polarized electrons or holes, injected into the semiconductor by ferromagnetic contacts. For this situation the monopolar spin relaxation is the decisive dynamical quantity, whose dependence on device parameters needs to be investigated.

In spite of recent progress, the injection of spin polarized carriers through heterocontacts remains a challenge that does not yet allow one to measure spin relaxation times. ${ }^{9,10}$ Therefore, monopolar optical spin orientation combined with the photogalvanic effects (PGE), which has been demonstrated for $n$ - and $p$-doped quantum well structures of different material compositions, ${ }^{11,12}$ is the method of choice to investi-

${ }^{a)}$ Electronic mail: petra.schneider@physik.uni-regensburg.de gate the spin dynamics of electrons or holes and avoid problems connected with electrical spin injection. It has been demonstrated $^{13}$ that linear and circular PGE show distinct saturation behavior with an increase in intensity of the exciting light which provide information about the spin relaxation time. Analysis of these data requires knowledge of the linear absorption coefficient for intersubband transitions, which is difficult to measure and is hence provided by realistic calculations in the self-consistent multiband envelope function approximation. ${ }^{14}$

We present here a detailed investigation of spin relaxation in rectangular $p$-type (113)-grown GaAs/AlGaAs quantum wells (QWs) of different widths $L_{W}$ and in a wide range of temperatures. This comprehensive experimental study of monopolar spin relaxation that depends on these two relevant system parameters, the width and the temperature, is accompanied by a theoretical analysis that relates the measured spin relaxation times to the D'yakonov-Perel' mechanism.

The article is organized as follows. First, we will present our samples and experimental technique and the results of measurements. Following that, we outline calculation of the absorption coefficient and using this calculation derive spin relaxation times. This is followed by a discussion of the dominant spin relaxation mechanism and the topic of selection rules.

\section{EXPERIMENT}

The experiments have been carried out on $p$-type (113) molecular beam epitaxy (MBE) grown GaAs/AlGaAs QWs with widths $L_{W}$ of 7, 10 and $15 \mathrm{~nm}$. In order to improve the sensitivity, multiple structures of 20 QWs were investigated. Samples with free carrier sheet densities $p_{s}$ of about 
(a)

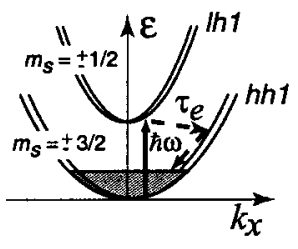

(b) circularly polarized

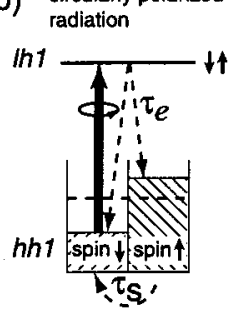

(c)

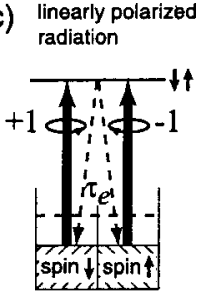

) linearly polarized

FIG. 1. Microscopic picture of spin sensitive bleaching: (a) direct hh1-lh1 optical transitions and (b) and (c) process of bleaching for two polarizations. Dashed arrows indicate energy $\left(\tau_{e}\right)$ and spin $\left(\tau_{s}\right)$ relaxation.

$10^{11} \mathrm{~cm}^{-2}$ and high mobility $\mu$ of around $10^{5} \mathrm{~cm}^{2} /(\mathrm{V} \mathrm{s})$ (at $4.2 \mathrm{~K}$ ) were studied from liquid helium temperature up to $140 \mathrm{~K}$. At the samples a pair of ohmic contacts is centered on opposite sample edges along the direction of $x \|[1 \overline{10}]$. As source of radiation a high power pulsed far-infrared (FIR) molecular laser, optically pumped by a TEA-CO $\mathrm{CO}_{2}$ laser, was used to deliver $100 \mathrm{~ns}$ pulses with intensity up to $1 \mathrm{MW} / \mathrm{cm}^{2}$ in the wavelength range of 76-148 $\mu \mathrm{m}$, thus providing direct intersubband transitions from the lowest heavy hole hh1 to the light hole lh1 subband. The radiation of the FIR laser is linearly polarized and a $\lambda / 4$ plate was used to generate circularly polarized radiation with polarization degree $P_{\text {circ }}$ $= \pm 1$ for right- and left-handed circularly polarized light.

The absorption of terahertz radiation by free carriers in QWs is weak due to their small thickness and is difficult to measure in transmission experiments. This is even worse in the case of bleaching at high power levels. Therefore, the nonlinear behavior of the absorption was investigated employing recently observed circular (CPGE) and linear photogalvanic effects (LPGE). ${ }^{11,12}$ Both CPGE and LPGE yield easy to measure electrical current in the $x$ direction. According to Ivchenko and Pikus ${ }^{15}$ the nonlinear absorption coefficient is proportional to the photogalvanic current $j_{x}$ normalized by the radiation intensity $I$. Thus, by choosing the degree of polarization, we obtain a photoresponse that corresponds to the absorption coefficient of circularly or linearly polarized radiation.

The investigated intensity dependence of the absorption coefficient $\alpha \propto j_{x} / I$ shows saturation with higher intensities for all samples used in our experiments. It is observed that saturation takes place for excitation with circularly polarized radiation at a lower level of intensity than excitation with linearly polarized radiation. The basic physics of this spin sensitive bleaching of absorption can be understood by looking at Fig. 1. Illuminating a $p$-type sample with FIR radiation of appropriate wavelength results in direct transitions between the heavy-hole hh1 and the light-hole lh1 subbands. This process selectively depopulates and populates spin states in hh1 and lh1 subbands. The absorption is proportional to the difference in population of the initial and final states. At high intensities the absorption decreases since the photoexcitation rate becomes comparable to the nonradiative relaxation rate back to the initial state. For $\mathrm{C}_{s}$ symmetry, which is relevant to our (113)-grown QWs, the selection rules for absorption at $\mathbf{k}$ close to zero are such that only one

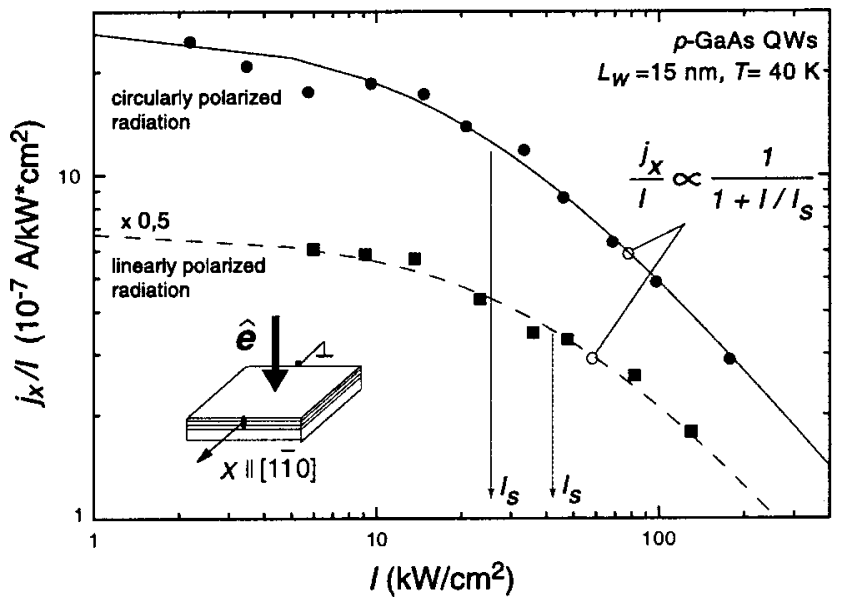

FIG. 2. CPGE and LPGE currents $j_{x}$ normalized by the intensity as a function of the intensity for circularly and linearly polarized radiation of $\lambda=148$ $\mu \mathrm{m}$ at $T=40 \mathrm{~K}$.

type of spin is involved in the absorption of circularly polarized light (a closer look at selection rules will be given at the end of this article). Thus absorption bleaching of circularly polarized radiation is governed by energy relaxation of photoexcited carriers and spin relaxation within the initial spinsplit subband [see Figs. 1(a) and 1(b)]. These processes are characterized by energy and spin relaxation times $\tau_{e}$ and $\tau_{s}$, respectively. We note that during energy relaxation to the initial state in hh1, the holes lose their photoinduced spin orientation due to rapid relaxation. ${ }^{16}$ Thus, spin orientation occurs in the initial subband hh1 only. In contrast to circularly polarized light, absorption of linearly polarized light is not spin selective and saturation is controlled by energy relaxation only [see Fig. 1(c)]. For $\tau_{s}>\tau_{e}$, bleaching of absorption becomes spin sensitive and the saturation intensity $I_{s}$ of circularly polarized radiation drops below the value of linear polarization as indicated in Fig. 2 by arrows. The saturation intensity is defined as the intensity at which $j_{x} / I$ is one half its unsaturated value at $I \rightarrow 0$.

Figure 3 presents the saturation intensities for different QW widths in the whole temperature range investigated. Note that saturation intensities $I_{s}$ for excitation with circularly polarized radiation (circles) are generally smaller than those for linearly polarized radiation (squares). A significant reduction of saturation intensity with a decrease in $L_{W}$ is observed and it indicates longer hole spin relaxation times for narrower QWs, which was shown theoretically in Ref. 16.

The nonlinear behavior of photogalvanic currents was analyzed in terms of excitation-relaxation kinetics taking into account both optical excitation and nonradiative relaxation processes. It was shown ${ }^{13}$ that the photocurrent $j_{\text {LPGE }}$, induced by linearly polarized radiation, is given by $j_{\mathrm{LPGE}} / I \propto\left(1+I / I_{s e}\right)^{-1}$, where $I_{s e}$ is the saturation intensity controlled by energy relaxation of the hole gas, whereas the photocurrent induced by circularly polarized radiation $j_{\mathrm{CPGE}} \propto I /\left[1+I\left(I_{s e}^{-1}+I_{s s}^{-1}\right)\right]$ in addition is controlled by spin relaxation by the term $I_{s s}=\hbar \omega p_{s} /\left(\alpha_{0} L_{W} \tau_{s}\right)$. Here $\alpha_{0}$ is the unsaturated absorption coefficient at low intensities. Thus the spin relaxation time $\tau_{s}$ is given by 

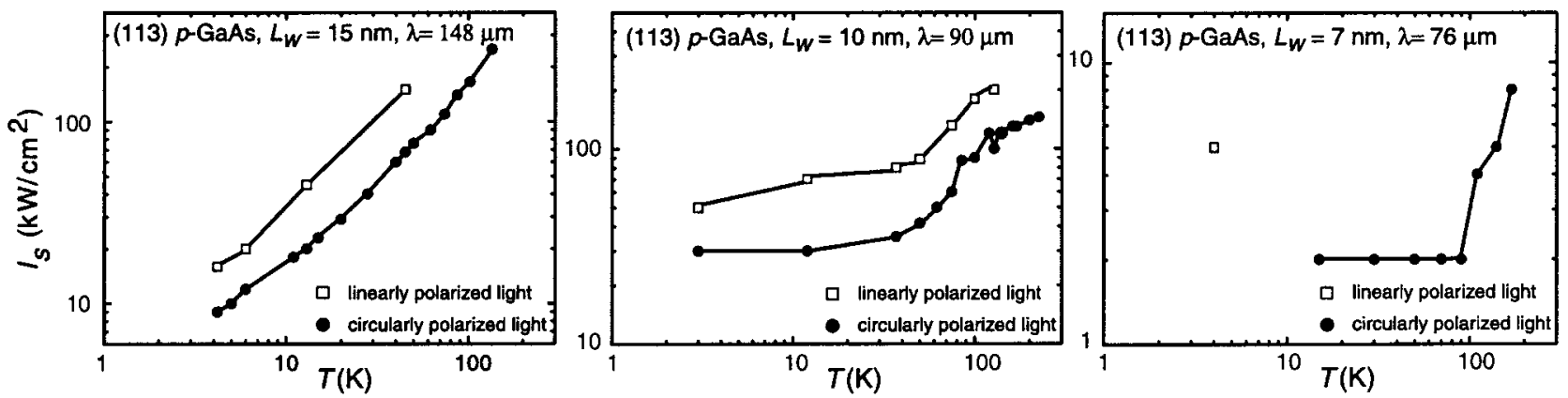

FIG. 3. Temperature dependence of the saturation intensities for various QW widths for linearly (open squares) and circularly (closed circles) polarized light. The thickness of the QWs decreases from left to right.

$$
\tau_{s}=\frac{\hbar \omega p_{s}}{\alpha_{0} L_{W} I_{s s}} .
$$

\section{ABSORPTION COEFFICIENT}

In order to obtain $\tau_{s}$ with this formula from the measured saturation intensities $I_{s s}$, the value of $\alpha_{0}$, not available from experiment, is determined theoretically. Calculations of the linear absorption coefficient $\alpha_{0}$ for intersubband transitions are based on the self-consistent multiband envelope function approximation (EFA) ${ }^{14}$ that takes into account the crystallographic orientation of the QW (here the [113] direction) and the doping profile. ${ }^{17}$ Calculations are performed using the Luttinger model of heavy and light hole states to obtain the hole subband dispersion $\epsilon_{i}(\mathbf{k})$ and eigenstates $|i, \mathbf{k}\rangle$ of hole subband $i$ and in-plane wave vector $\mathbf{k}$. For direct (electrical dipole) transitions between subbands $i$ and $j$ the contribution to the absorption coefficient $\alpha_{i \rightarrow j}(\omega)$ as a function of excitation energy $\hbar \omega$ is then given by ${ }^{18}$

$$
\begin{aligned}
\alpha_{i \rightarrow j}(\omega)= & \frac{e^{2}}{4 \pi \epsilon_{0} \omega c n L_{W}} \int \mathrm{d}^{2} k|\langle j, \mathbf{k}|\mathbf{e} \cdot \hat{\mathbf{v}}(\mathbf{k})| i, \mathbf{k}\rangle|^{2} \\
& \times\left[f_{j}(\mathbf{k})-f_{i}(\mathbf{k})\right] \frac{\mathbf{e}^{-\left[\epsilon_{j}(\mathbf{k})-\epsilon_{i}(\mathbf{k})-\hbar \omega\right]^{2} / \Gamma^{2}}}{\sqrt{\pi} \Gamma},
\end{aligned}
$$

where $\mathbf{e}$ is the light polarization vector, $n$ is the refractive index, $\epsilon_{0}$ is the free-space permittivity, $f_{i}(\mathbf{k})$ is the Fermi distribution function in the subband $i$, and $\Gamma$ is a phenomeno- logical parameter to account for level broadening due to scattering. In EFA, the velocity $\hat{\mathbf{v}}(\mathbf{k})$ is a matrix operator expressed as the gradient in $\mathbf{k}$-space of the Luttinger Hamiltonian. Its matrix elements are calculated from the EFA wave functions.

Following this scheme we calculate the absorption coefficient $\alpha_{0}(\omega)=\Sigma_{i j} \alpha_{i \rightarrow j}(\omega)$. The absorption spectrum for the system with $L_{W}=7 \mathrm{~nm}$ is shown in Fig. 4(a). At low temperatures two pronounced peaks evolve, which correspond to transitions from the lowest (spin split) hole subband to the second and third subbands, respectively. Figure 4(b) shows the temperature dependence (due to the Fermi distribution function) of $\alpha_{0}$ at respective excitation energies for the different samples. The calculated values of $\alpha_{0}$ are used to convert the measured saturation intensities $I_{s s}$ according to Eq. (1) into spin relaxation times $\tau_{s}$.

The resulting hole spin relaxation times that depend on the temperature are shown in Fig. 5 for QWs of different widths. Our measurements show longer hole spin relaxation times for narrower QWs. Note the different behavior of the spin relaxation times with the temperature for different $\mathrm{QW}$ widths. It is worth mentioning that at high temperatures doubling of the QW width decreases $\tau_{s}$ by almost two orders of magnitude. Compared to the values given in Ref. 13 (for $L_{W}=15 \mathrm{~nm}$ ), where $\alpha_{0}$ was derived from Ref. 18, we obtain here smaller $\tau_{s}$ at higher temperatures due to a more realistic theoretical model for the calculation of $\alpha_{0}$.
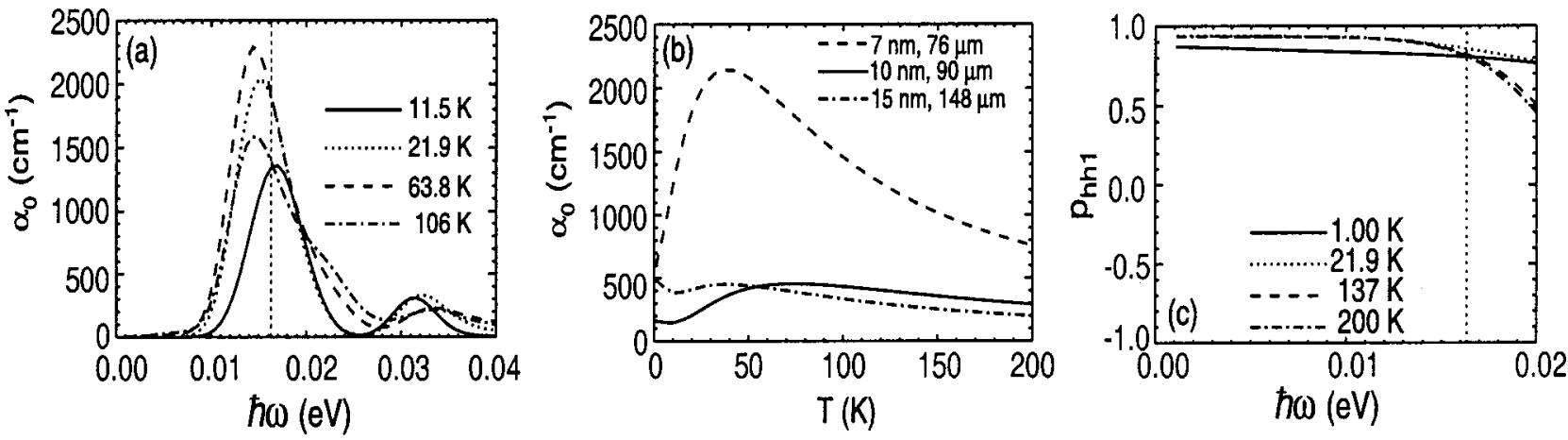

FIG. 4. (a) Calculated absorption coefficient $\alpha_{0}$ for a QW with $L_{W}=7 \mathrm{~nm}$ as a function of photon energy $\hbar \omega$ for various temperatures $T$ and (b) as a function of $T$ for various QW widths with $\hbar \omega$ corresponding to the energy of the exciting laser light. (c) Hole spin orientation efficiency $p_{\text {hh1 }}$ as a function of $\hbar \omega$ for different $T, L_{W}=7 \mathrm{~nm}$, and right handed circular polarization. All calculations were performed for carrier density $p_{s}$ of about $2 \times 10^{11} \mathrm{~cm}^{-2}$ and broadening $\Gamma=2.47 \mathrm{meV}$. 


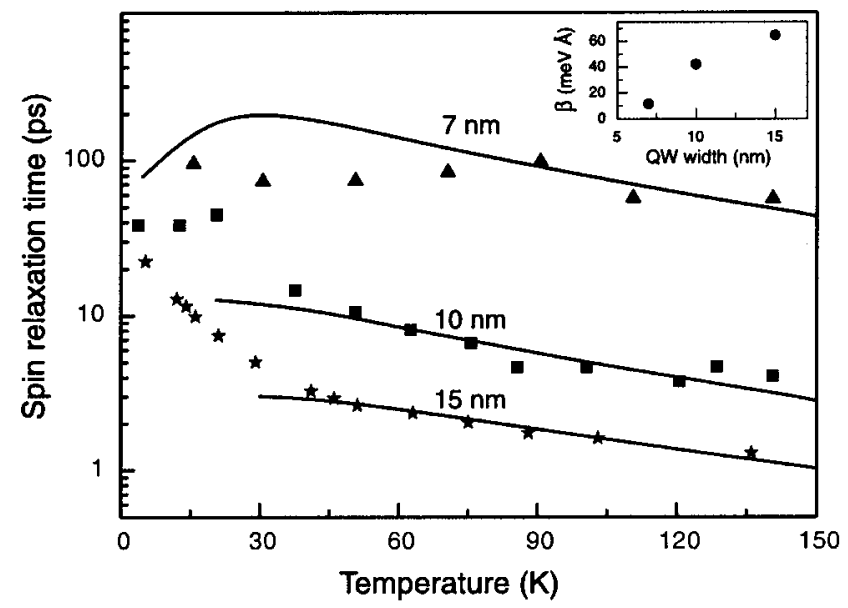

FIG. 5. Spin relaxation times of holes for three different widths of (113)grown GaAs/AlGaAs QWs as a function of the temperature. The solid lines show a fit according to the D'yakonov-Perel' relaxation mechanism. The inset shows hole spin-splitting parameter $\beta$ obtained from the fit.

\section{SPIN RELAXATION MECHANISM}

In order to understand the mechanism that governs spin relaxation, we consider the ratio of momentum $\tau_{p}$ and spin $\tau_{s}$ relaxation times at $T=4.2 \mathrm{~K}$ presented in Table I. In the $p$-doped QWs, studied here, there are two possible routes to hole spin relaxation: the Elliot-Yafet mechanism or the D'yakonov-Perel' mechanism. In the first, spin is lost during scattering. However the ratio $\tau_{p} / \tau_{s}$ for holes, where $\tau_{p}$ is determined from mobility measurements, has strong dependence on the QW width $\left(\sim L_{W}^{6}\right)$ for scattering due to impurity or interface microroughness. Note that for calculation of the spin relaxation time we do not take into account phonon scattering because most of the experimental data are for the range of low temperatures where phonon scattering processes play an unimportant role. In addition, $\tau_{p}$ is of the same order as $\tau_{s}$ for the two wider QWs which contradicts the main idea of the Elliot-Yafet mechanism. Another possibility is Elliot-Yafet spin relaxation controlled by holehole collisions, but for this mechanism asymmetry of the QW heteropotential is needed. ${ }^{19}$

We conclude that the Elliot-Yafet mechanism is unimportant in the structures under study, since the experiment shows too weak dependence for $\tau_{p} / \tau_{s}$ on the QW width. The above experimental results suggest much longer spin relaxation times for the given mobilities than those expected for the Elliot-Yafet mechanism. The spin relaxation time at helium temperature according to the Elliot-Yafet mechanism can be estimated as

TABLE I. Momentum relaxation times $\tau_{p}$ (determined from the mobility) and ratios $\tau_{p} / \tau_{s}$ for different QW widths at $4.2 \mathrm{~K}$.

\begin{tabular}{ccl}
\hline \hline $\begin{array}{c}\text { QW width } \\
(\mathrm{nm})\end{array}$ & $\begin{array}{c}\tau_{p} \\
(\mathrm{ps})\end{array}$ & $\tau_{p} / \tau_{s}$ \\
\hline 7 & 9.5 & 0.1 \\
10 & 25 & 0.64 \\
15 & 38 & 1.73 \\
\hline \hline
\end{tabular}

$$
\tau_{s} \approx \tau_{p}\left(\frac{k_{\mathrm{F}} L_{W}}{\pi}\right)^{-6},
$$

where $\mathbf{k}_{F}$ is the Fermi wave vector. This yields $\tau_{s} \approx 5 \times 10^{5} \mathrm{ps}$ which is three orders of magnitude larger than measured values. Therefore, the main mechanism of hole spin relaxation is the D'yakonov-Perel' mechanism: ${ }^{20}$ hole spin is lost between scattering events. For this mechanism, the spin relaxation rate is given by

$$
\frac{1}{\tau_{s}}=\left(\frac{\beta}{\hbar}\right)^{2} k_{\mathrm{F}}^{2} \tau^{*},
$$

where $\beta$ is the spin-splitting coefficient of the $\mathbf{k}$-linear terms in the Hamiltonian, yielding

$$
E_{3 / 2}(k)-E_{-3 / 2}(k)=2 \beta k \text {. }
$$

The time $\tau^{*}$ is the microscopic scattering time which has contributions from both momentum scattering and carriercarrier collisions. ${ }^{21}$ We have calculated the hole-hole scattering time governing the D'yakonov-Perel' spin relaxation mechanism by solving the quantum kinetic equation for the hole pseudospin density matrix similar to in Ref. 22. Our calculation shows that the hole-hole scattering time is shorter than $\tau_{p}$ at $4.2 \mathrm{~K}$. We believe that in the relevant temperature range $\tau_{p}$ does not change significantly. Therefore, hole-hole scattering controls D'yakonov-Perel' spin relaxation in the whole temperature range.

Figure 5 presents spin relaxation times extracted from experiment (points) together with a theoretical fit using Eq. (3) (solid lines), showing a good agreement between theory and experiment. The discrepancy at low lattice temperatures may be attributed to the fact that the hole gas is not in equilibrium due to optical pumping. This case requires special theoretical treatment.

In the inset in Fig. 5 the hole spin-splitting parameter $\beta$ obtained from the fit is plotted as a function of the QW width. The corresponding spin splitting is equal to $0.17,0.68$, and $1.32 \mathrm{meV}$ for QW widths of 7, 10, and $15 \mathrm{~nm}$, respectively. This order of magnitude agrees with hole spin splitting obtained from multiband calculations. ${ }^{23}$ The parameter $\beta$ increases with the $\mathrm{QW}$ width. This is a specific feature of two-dimensional hole systems where spin splitting is determined by heavy-light hole mixing, which is stronger in wider QWs. ${ }^{24}$

\section{SELECTION RULES AND SPIN ORIENTATION}

For the definition of $I_{s s}$ we assumed that the spin selection rules are fully satisfied at the transition energy. This is the case for optical transitions that occur close to $\mathbf{k}=0$ in (001)-grown systems. ${ }^{25}$ However, in (113)-grown systems, heavy-hole and light-hole subbands are strongly mixed, even at $\mathbf{k}=0$. This reduces the strength of the selection rules and therefore the efficiency of the spin orientation. Mixing can be taken into account by means of a multiplicative factor in $I_{s s}$, which increases the saturation intensity at constant spin relaxation time. ${ }^{26}$

The lowest subband, which for (001)-grown systems is purely heavy hole $\left(m_{s}= \pm 3 / 2\right)$ at $\mathbf{k}=0$, has for growth direction [113] an admixture of about 10\% light hole spinor 
components $\left(m_{s}= \pm 1 / 2\right) .{ }^{27}$ This admixture is sufficiently small to justify subband labeling according to the dominant spinor component at $\mathbf{k}=0$.

Strict selection rules for intersubband transitions between hole subbands only exist for some idealized limits (e.g., spherical approximation for the Luttinger Hamiltonian or growth directions of high symmetry and $\mathbf{k}=0$ ). However, assuming a symmetrically doped (113)-grown QW, the lowest hh and lh subband states (hh1 and lh1, respectively) have even parity at $\mathbf{k}=0$ and no transition between hh1 and lh1 is possible, since the velocity operator projected on the light polarization direction $\hat{\mathbf{v}} \cdot \mathbf{e}$ couples only states of different parity. Therefore a strictly valid selection rule cannot be obtained and a more quantitative discussion of the relative weight of possible transitions is necessary. For $\mathbf{k}$ small enough to ensure that the admixture of odd parity spinor components is negligible, only contributions in $\hat{\mathbf{v}} \cdot \mathbf{e}$ linear in $\mathbf{k}$ are considered.

A more detailed analysis gives the following results: The spin-conserving transitions $\mathrm{hh} 1 \uparrow \rightarrow \mathrm{lh} 1 \uparrow$ and $\mathrm{hh} 1 \downarrow \rightarrow \mathrm{lh} 1 \downarrow$ are much weaker than the corresponding spin-flip transitions hh $1 \uparrow \rightarrow \operatorname{lh} 1 \downarrow$ and hh $1 \downarrow \rightarrow \mathrm{lh} 1 \uparrow$. Depending on the left/right circular polarization of the excitation light, one of the spin-flip transitions is dominant. To investigate the hole spin orientation, we also performed numerical calculation of $\alpha_{i \rightarrow j}$ for excitation with right-hand circularly polarized light. We found that the transition hh1 $\downarrow \rightarrow \operatorname{lh} 1 \uparrow$ is far more probable than all other transitions. This is quantitatively described by the heavy-hole spin polarization efficiency,

$$
p_{\mathrm{hh} 1}=\frac{\sum_{i} \alpha_{\mathrm{hh} 1 \downarrow \rightarrow i}-\alpha_{\mathrm{hh} 1 \uparrow \rightarrow i}}{\sum_{i} \alpha_{\mathrm{hh} 1 \downarrow \rightarrow i}+\alpha_{\mathrm{hh} 1 \uparrow \rightarrow i}},
$$

where summation is performed over all subbands. If $p_{\text {hh1 }}$ is $+1(-1)$ excitation leaves only heavy holes belonging to the up (down) branch of dispersion in the hh1 subband. In our case, $p_{\text {hh1 }}$ is around $80 \%$ at the laser excitation energy and almost independent of the temperature [Fig. 4(c)]. Therefore one can neglect effects due to incomplete spin orientation, as assumed in the above analysis.

In conclusion our experimental results demonstrate strong dependence of the hole spin relaxation times on the width of the quantum well. With wider QWs, the spin relaxation times become much shorter. At high temperatures, doubling of the QW width results in a change of magnitude of two orders. A comparison of theoretical calculations and quantitative experimental results shows that the D'yakonovPerel' mechanism controlled by hole-hole collisions dominates the spin relaxation process.

\section{ACKNOWLEDGMENTS}

The authors thank E. L. Ivchenko for helpful discussions and fruitful comments. Financial support from the Deutsche Forschungsgemeinschaft, the Russian Foundation for Basic Research, "Dynasty" Foundation, ICFPM, and INTAS is gratefully acknowledged.

${ }^{1}$ Semiconductor Sprintronics and Quantum Computation, edited by D. D. Awshalom, D. Loss, and N. Samarth (Springer Verlag, Berlin, 2002).

${ }^{2}$ G. E. Pikus and A. N. Titkov, in Optical Orientation, edited by F. Meier and B. P. Zakharchenya (Elsevier, Amsterdam, 1984).

${ }^{3}$ T. C. Damen, L. Viña, J. E. Cunningham, J. Shah, and L. J. Sham, Phys. Rev. Lett. 67, 3432 (1991).

${ }^{4}$ M. Kohl, M. R. Freeman, D. D. Awschalom, and J. M. Hong, Phys. Rev. B 44, 5923 (1991).

${ }^{5}$ A. Tackeuchi, Y. Nishikawa, and O. Wada, Appl. Phys. Lett. 68, 797 (1996)

${ }^{6}$ R. Terauchi, Y. Ohno, T. Adachi, A. Sato, F. Matsukura, A. Tackeuchi, and H. Ohno, Jpn. J. Appl. Phys., Part 1 38, 2549 (1999).

${ }^{7}$ G. L. Bir, A. G. Aronov, and G. E. Pikus, Zh. Éksp. Teor. Fiz. 69, 1382 (1975), [Sov. Phys. JETP 42, 705 (1976)].

${ }^{8}$ S. Datta and B. Das, Appl. Phys. Lett. 56, 665 (1990).

${ }^{9}$ Y. Ohno, D. K. Young, B. Beschoten, F. Matsukura, H. Ohno, and D. D. Awshalom, Nature (London) 402, 790 (1999).

${ }^{10}$ G. Schmidt, D. Ferrand, L. W. Molenkamp, A. T. Filip, and B. J. van Wees, Phys. Rev. B 62, R4790 (2000).

${ }^{11}$ S. D. Ganichev, E. L. Ivchenko, H. Ketterl, W. Prettl, and L. E. Vorobjev, Appl. Phys. Lett. 77, 3146 (2000).

${ }^{12}$ S. D. Ganichev, E. L. Ivchenko, S. N. Danilov, J. Eroms, W. Wegscheider, D. Weiss, and W. Prettl, Phys. Rev. Lett. 86, 4358 (2001).

${ }^{13}$ S. D. Ganichev, S. N. Danilov, V. V. Bel'kov, E. L. Ivchenko, M. Bichler, W. Wegscheider, D. Weiss, and W. Prettl, Phys. Rev. Lett. 88, 057401 (2002).

${ }^{14}$ R. Winkler and U. Rössler, Phys. Rev. B 48, 8918 (1993).

${ }^{15}$ E. L. Ivchenko and G. E. Pikus, Superlattices and Other Heterostructures, Symmetry and Optical Phenomena (Springer, Berlin, 1997).

${ }^{16}$ R. Ferreira and G. Bastard, Phys. Rev. B 43, 9687 (1991).

${ }^{17} \mathrm{In}$ accordance with the growth parameters of the samples, we assumed an acceptor concentration of $1 \times 10^{16} \mathrm{~cm}^{-3}$ in the barriers and spacer width of $70 \AA$ ( $45 \AA$ ) on the left (right) side of the well. The values of the band parameters are identical to those given in L. Wissinger, U. Rössler, B. Jusserand, and D. Richards, Phys. Rev. B 58, 15375 (1998).

${ }^{18}$ L. E. Vorobjev, D. V. Donetskii, and L. E. Golub, Pis'ma Zh. Éksp. Teor. Fiz. 63, 928 (1996) [JETP Lett. 63, 977 (1996)].

${ }^{19}$ M. M. Glazov and E. L. Ivchenko (unpublished).

${ }^{20}$ G. Bastard and R. Ferreira, Europhys. Lett. 23, 439 (1993).

${ }^{21}$ M. M. Glazov and E. L. Ivchenko, Pis'ma Zh. Éksp. Teor. Fiz. 75, 476 (2002) [JETP Lett. 75, 403 (2002)].

${ }^{22}$ M. M. Glazov, E. L. Ivchenko, M. A. Brand, O. Z. Karimov, and R. T. Harley, Proceedings of the 11th International Symposium Nanostructures: Physics and Technology, St. Petersburg, 2003, p. 273; cond-mat/0305260.

${ }^{23}$ R. Winkler, Phys. Rev. B 62, 4245 (2000).

${ }^{24}$ R. Winkler, H. Noh, E. Tutuc, and M. Shayegan, Phys. Rev. B 65, 155303 (2002).

${ }^{25}$ S. Jorda and U. Rössler, Superlattices Microstruct. 8, 481 (1990).

${ }^{26}$ S. D. Ganichev, V. V. Bel'kov, S. N. Danilov, E. L. Ivchenko, H. Ketterl, L. E. Vorobjev, M. Bichler, W. Wegscheider, and W. Prettl, Physica E (Amsterdam) 10, 52 (2001).

${ }^{27}$ R. Winkler and A. I. Nesvizhskii, Phys. Rev. B 53, 9984 (1996). 